\title{
„Strafe muss sein!“ \\ Plädoyer für die Einrichtung von Sonderdezernaten „Häusliche Gewalt“ bei den Staatsanwaltschaften
}

\begin{abstract}
Sabine Kräuter-Stockton
Vorsitzende des Landesverbands Saarland im djb, Vorsitzende der AG „Straftaten gegen Ältere“ und Mitglied der Fachkommission „Gewalt gegen Frauen und Kinder" in der Kommission Strafrecht des djb; Staatsanwältin/Gruppenleiterin, Saarbrücken
\end{abstract}

Die jüngst veröffentlichte Auswertung der djb-Länderumfrage zum zehnjährigen Bestehen des Gewaltschutzgesetzes hat es an den Tag gebracht: Anders als erwartet hat sich der noch vor einigen Jahren beobachtete Trend zu immer mehr Sonderdezernaten „Häusliche Gewalt“ bei den Staatsanwaltschaften nicht fortgesetzt. Von einer bundesweit flächendeckenden Einrichtung sind wir bedauerlicherweise noch weit entfernt. Vielmehr scheinen es sich viele Behörden nach dem Ausklingen des ersten Schwungs im einmal erreichten Zustand bequem gemacht zu haben und nicht an einer weiteren Verbesserung ihrer Struktur interessiert zu sein.

Dabei besteht heute in der Fachwelt breite Einigkeit darüber, dass eine konsequente Strafverfolgung wesentlicher Bestandteil einer effektiven Bekämpfung häuslicher Gewalt ist. Da reicht es nicht, dass 1994 durch Beschluss der Justizministerkonferenz die Richtlinien für das Straf- und Bußgeldverfahren (RiStBV) geändert wurden ${ }^{1}$, damit künftig möglichst wenig Opfer auf den Weg der Privatklage verwiesen werden. Werden Verfahren häuslicher Gewalt in allgemeinen Abteilungen der Staatsanwaltschaften bearbeitet, gehen sie nämlich - ohne das in Sonderdezernaten vorhandene spezielle Knowhow - in der Masse unter und werden weitgehend doch durch Einstellungen bzw. Privatklageverweisungen erledigt.

Was das bedeutet, ist den Fachleuten schon lange klar: Die Einstellung eines Strafverfahrens verfestigt in der Regel die Strukturen einer Gewaltbeziehung, weil sie sowohl dem Täter ${ }^{2}$ wie dem Opfer - wie auch übrigens der Öffentlichkeit - signalisiert, dass die Tat kein sanktionswürdiges Unrecht darstellt, und dass der Täter sein Verhalten ungestraft fortsetzen kann. Worin besteht nun das „Know-how“ in einem Sonderdezernat „Häusliche Gewalt“, wie sollte der Zuschnitt des Dezernats aussehen?

Wenn in dem Sonderdezernat nicht nur Vergehen wie Körperverletzungen, Nötigungen, Bedrohungen etc., sondern auch Verbrechen wie Sexualdelikte 3 bearbeitet werden, vermeidet dies zuständigkeitsbedingte Abgaben zwischen verschiedenen Dezernaten und damit Zeit- und Ressourcenverluste. Umgekehrt ist die Folge eines zu engen Zuschnitts der Sonderdezernate nur auf minder schwere Delikte, dass Reibungsverluste entstehen, wenn sich im Rahmen der Ermittlungen herausstellt, dass daneben auch noch schwere Straftaten wie Vergewaltigungen begangen wurden. Wegen des Dezernatszuschnitts muss dann entweder das Verfahren wegen des Sexualdelikts abgetrennt und gesondert verfolgt werden - was gar keinen Sinn macht: doppelte Ermittlungen im selben Zusammenhang! - oder es muss das gesamte Verfahren, das zuvor unter dem Aspekt „Körperverletzungsdelikte im Rahmen häuslicher Gewalt" mit Sonderzuständigkeit bearbeitet wurde, nunmehr wegen des Verbrechensvorwurfs an ein anderes hierfür zuständiges (aber auf häusliche Gewalt nicht spezialisiertes) Dezernat abgegeben werden.

In den Sonderdezernaten „Häusliche Gewalt“ haben sich nun - Sinn und Zweck jeder Spezialisierung - besonders erfolgreiche Vorgehensweisen durchgesetzt. Dies ist auch notwendig, denn Opfer von Partnergewalt verhalten sich ganz anders als Geschädigte sonstiger Delikte, und dieser Herausforderung für die Ermittlungen gilt es vorausschauend zu begegnen. Wem im Kaufhaus der Geldbeutel gestohlen wurde oder wer bei einem Internetkauf betrogen wurde, der arbeitet in der Regel bereitwillig mit, um der Polizei die/den Täter/in und die Tat zu beschreiben und alles dazu beizutragen, dass die/der Täterin überführt wird. Das Opfer einer Straftat ist normalerweise unser Hauptbeweismittel. Nicht so die Opfer von Partnergewalt. Sie lehnen meist nicht nur die Mithilfe an der Überführung des Täters ab, sondern arbeiten sogar teilweise aktiv gegen die Ermittlungen, sie machen keine Aussage oder lügen: die Verletzungen seien durch Dritte verursacht oder selbst beigefügt. Das bedeutet aber nicht, dass Strafverfolgung hier nicht nötig wäre, im Gegenteil: Es zeigt vielmehr, dass Ermittlungen und Strafverfolgung erst recht angezeigt sind.

\section{$1 \quad$ Nr. 86 RiStBV:}

„(1) Sobald der Staatsanwalt von einer Straftat erfährt, die mit der Privatklage verfolgt werden kann, prüft er, ob ein öffentliches Interesse an der Verfolgung von Amts wegen besteht."

„(2) Ein öffentliches Interesse wird in der Regel vorliegen, wenn (...). (...) kann ein öffentliches Interesse auch dann vorliegen, wenn dem Verletzten wegen seiner persönlichen Beziehung zum Täter nicht zugemutet werden kann, die Privatklage zu erheben und die Strafverfolgung ein gegenwärtiges Anliegen der Allgemeinheit ist." Nr. 233 RiStBV:

„Das öffentliche Interesse an der Verfolgung von Körperverletzungen ist vor allem dann zu bejahen, wenn (...). Dies gilt auch, wenn die Körperverletzung in einer engen Lebensgemeinschaft begangen wurde;(...)."

Nr. 234 Abs. 1 RiStBV:

„(1) Ein besonderes öffentliches Interesse an der Verfolgung von Körperverletzungen ( $\$ 230$ Abs. 1 Satz 1 StGB) wird namentlich dann anzunehmen sein, wenn (...) oder dem Opfer wegen seiner persönlichen Beziehung zum Täter nicht zugemutet werden kann, Strafantrag zu stellen, und die Strafverfolgung ein gegenwärtiges Anliegen der Allgemeinheit ist.

2 In der überwiegenden Mehrheit der Fälle häuslicher Gewalt ist der Täter männlich und das Opfer weiblich, so dass hier von „der Täter" und „die Geschädigte“ gesprochen wird.

3 Sexualverbrechen werden bekanntermaßen häufiger im Rahmen von Beziehungen oder Bekanntschaften begangen als durch fremde Täter. 
Um den Nachweis zu führen, muss der Sachverhalt - wenn das klassische Hauptbeweismittel fehlt - minutiös untersucht und analysiert werden, damit kein noch so geringer Hinweis übersehen wird. Besonders gründliche polizeiliche Tatortaufnahme vor Ort mit sorgfältiger Erhebung und Dokumentation aller in Frage kommender Beweise muss in Fällen von Partnergewalt die Regel sein. Hier ist die Staatsanwaltschaft mehr als üblich gefragt, die polizeilichen Ermittlungen konkret zu leiten und zu kontrollieren. So wird nicht selten die Akte von der Staatsanwaltschaft zurück an die Polizeidienststelle geschickt mit dem Auftrag, Beweiserhebungen nachzuholen, einen Notruf zu sichern, eine Zeugenvernehmung nachzubessern.

Es kann auch angezeigt sein, das Opfer nicht wie üblich polizeilich, sondern staatsanwaltschaftlich zu vernehmen. Zeug/inn/en sind ja nicht verpflichtet, bei der Polizei auszusagen, so dass viele Geschädigte von Partnergewalt sich auf die polizeiliche Vorladung zur Zeugenvernehmung gar nicht erst melden. Von der Staatsanwaltschaft kann eine Zeugin oder ein Zeuge dagegen durch Vorführung und Ordnungsgeld zum Erscheinen und, in Grenzen, zur Aussage gezwungen werden. Der sonst übliche Rückschluss aus dem Nichterscheinen einer/eines Geschädigten bei der Polizei - sie/er hat offensichtlich kein Interesse, also war es wohl nicht so schlimm - kann bei häuslicher Gewalt naturgemäß nicht gelten. Wohnen Täter und Opfer zusammen, so ist schon fraglich, ob das Opfer die Vorladung zur Polizei überhaupt erhalten hat, hat doch der Täter die besten Zugriffsmöglichkeiten auf den Brief mit der Ladung. Meist aber ist die Einstellung der Geschädigten dafür verantwortlich, dass sie nicht zum Termin kommt: Sie hofft auf Besserung des Täters und möchte nicht, dass er bestraft wird, oder sie hat - oft berechtigte - Angst. ${ }^{4}$ Im Rahmen einer staatsanwaltschaftlichen Vernehmung kann nun zumindest versucht werden, sie doch zur Aussage zu motivieren und sie dabei auf Beratungsstellen hinzuweisen. Manche Geschädigte bitten bei der Staatsanwaltschaft aus Angst vor Repressalien des Täters darum, dass im Protokoll der Zeugenvernehmung festgehalten werde, dass ihnen erst mit Zwangsmitteln gedroht werden musste, bevor sie eine Aussage machten. Ihnen ist klar, dass der Beschuldigte später über seinen Verteidiger erfahren wird, wie sie sich in der Vernehmung geäußert haben.

Wesentlich ist, dass das Opfer möglichst zeitnah, umfassend und professionell zur Tat vernommen wird. Eine zeitnahe Vernehmung ist deswegen geboten, weil viele Geschädigte zwar in den ersten Tagen unter dem Eindruck der Gewalttat noch aussagebereit sind, sich aber dann oft nach einiger Zeit entscheiden, den Täter doch nicht zu belasten. Die Informationen, die sich die Ermittlungsbehörden zu Beginn verschaffen können, sind ein wichtiger Baustein für die Planung des weiteren Vorgehens. Durch Hinweise auf weitere als Zeug/inn/en in Betracht kommende Personen lassen sich die Ermittlungen später auch dann noch fortsetzen, wenn die Geschädigte irgendwann nicht mehr aussagebereit ist. ${ }^{5}$ Auch kann eine frühe Vernehmung der Geschädigten Grundlage für eine realistische Gefährdungseinschätzung sein, an die sich polizeiliche oder staatsanwaltschaftliche Eilmaßnahmen wie eine Wohnungsverweisung oder ein Haftbefehlsantrag anschließen können.

Wichtig ist, dass die Vernehmungsperson die Vernehmung gut vorbereitet und auf alle Tatbestandsmerkmale des in Frage kommenden Paragrafen eingeht. So wird nicht selten in der Vernehmung zum Vorwurf einer Körperverletzung vergessen, das zweite Tatbestandsmerkmal neben der Tathandlung, nämlich die Folgen der Handlung für die Geschädigte, abzuklären. Wurde sie verletzt? Wenn ja: in welcher Weise und wo? Hatte sie, falls sie nicht verletzt wurde, Schmerzen, die mehr als unerheblich waren? Zeug/inn/en und Geschädigte beschreiben von sich aus meist nur die Handlungen des Täters: Er schlug, trat, würgte, aber die blauen Flecken, die blutende Nase, der ausgerenkte Daumen werden im Bericht oft vergessen. Hier muss die Vernehmungsperson nachhaken. Wenn die Folgen der Tathandlung nicht festgestellt sind, ist der Nachweis einer Körperverletzung nicht geführt.

Selbstverständlich muss die Vernehmung auch umfassend sein und darf nicht allein auf die Anlasstat abstellen. Wenn die Vernehmungsperson über das Hintergrundwissen verfügt, dass häusliche Gewalt in der Regel kein einmaliges Delikt ist, wird sie die Geschädigte nicht nur zu der aktuellen Tat befragen, sondern auch dazu, ob es in der Vergangenheit zu weiteren Taten kam. Wenn die Geschädigte hier weitere strafrechtlich relevante Vorfälle beschreibt, dann werden entsprechend dem Legalitätsprinzip ${ }^{6}$ die Ermittlungen auch auf diese weiteren Taten ausgedehnt.

$\mathrm{Zu}$ einer professionellen Vernehmung gehört auch, dass die Vernehmungsperson während der Vernehmung sorgfältig neutral bleibt. Dem Opfer darf einerseits nicht unterschwellig vermittelt werden, dass es ja eigentlich „selbst schuld“ sei, weil es sich nicht schon längst von dem Täter getrennt hat. Wenn die Vernehmungsperson andererseits vehement gegen den Täter Position bezieht, nimmt die Geschädigte unter Umständen den Täter - wie sie es schon so oft getan hat - in Schutz.

Die meisten Geschädigten bei häuslicher Gewalt besitzen entweder als Ehepartnerin oder als Verlobte ${ }^{7}$ ein Zeugnisver-

4 Zu Beginn sind die Opfer oft noch voller Hoffnung, dass es sich nur um einen „Ausrutscher" des Täters handelte und dass er sich jetzt wieder zu dem liebevollen Mann zurückverwandeln wird, der er früher war. Wenn die Gewalt sich dann aber wiederholt und steigert, ist es die Angst, die sie schweigen lässt: Sie wissen jetzt, wozu der Täter fähig ist und fürchten, dass er sie oder die Kinder umbringt, wenn sie gegen ihn Aussage machen.

5 Wenn die Geschädigte in ihrer ersten Vernehmung von einem Vorfall berichtet, nach dem sie sich zu ihrer besten Freundin geflüchtet hat, und die Vernehmungsperson (Polizei oder Staatsanwaltschaft) hakt hier ein und erfragt die Personalien der Freundin, dann kann diese auch bei späterer Zeugnisverweigerung der Geschädigten dazu vernommen werden. Aus ihren Angaben dazu, was die Geschädigte über die Tat erzählte und welche Verletzungen zu sehen waren, kann der Nachweis einer Körperverletzung geführt werden, auch ohne Aussage der Geschädigten selbst.

6 Das Legalitätsprinzip besagt, dass die Strafverfolgungsbehörden zur Einleitung eines Ermittlungsverfahrens verpflichtet sind, wenn sie Kenntnis von einer Straftat erhalten.

7 Ein behauptetes Verlöbnis ist - falls nicht eine/ $r$ der beiden angeblichen Verlobten mit einer dritten Person verheiratet ist - praktisch nicht zu widerlegen. 
weigerungsrecht gemäß $\mathbb{S} 52$ StPO. Wenn sie sich hierauf berufen, dann bedeutet das nicht nur, dass sie nicht zur Aussage gezwungen werden können. Die Inanspruchnahme des Zeugnisverweigerungsrechts bedeutet gleichzeitig gemäß $\mathbb{S} 252$ StPO auch, dass ein Urteil sich nicht auf eine frühere Aussage stützen darf, die die Geschädigte im Ermittlungsverfahren gegenüber Staatsanwaltschaft oder Polizei ${ }^{8}$ gemacht hat - ganz gleich, wie detailliert und glaubhaft die Aussage und wie umfassend damals die Belehrung war.

Unser Prozessrecht sieht dennoch Möglichkeiten vor, ausnahmsweise frühere Angaben von zeugnisverweigerungsberechtigten Zeugen trotz späterer Zeugnisverweigerung zu verwerten. Die Verwertung einer ermittlungsrichterlichen Zeugenvernehmung ist beispielsweise erlaubt. Das Urteil darf sich darauf stützen, was die/der in der Hauptverhandlung als Zeugin/Zeuge vernommene Ermittlungsrichter/in von ihrer/ seiner Vernehmung der Zeugin noch erinnert. Dies ist jedoch nicht immer erfolgreich, wenn etwa erst zu einem so späten Zeitpunkt ein Termin für die ermittlungsrichterliche Vernehmung gefunden wird, dass die Zeugin sich in der Zwischenzeit schon entschieden hat, doch nicht gegen den Täter auszusagen. Auch kommt es vor, dass die/der Ermittlungsrichter/in sich in der Hauptverhandlung nicht mehr konkret an die Inhalte der vor Monaten durchgeführten Vernehmung erinnern kann. Eine andere Möglichkeit, Angaben von zeugnisverweigerungsberechtigten Zeug/inn/en in die Hauptverhandlung verwertbar einzuführen, besteht in der sogenannten Spontanäußerung. Hier handelt es sich um Angaben, die die Geschädigte nicht als „Aussage“ im Rahmen eines Ermittlungsverfahrens gemacht hat, sondern während eines Hilfeersuchens gegenüber der Polizei. In Betracht kommt da etwa der Anruf vom Mobiltelefon aus bei der Polizei: „Hilfe, ich habe mich im Badezimmer eingesperrt, mein Mann hat mich eben geschlagen ...". Hier handelt es sich nicht um eine Aussage im Sinn der StPO, sondern um ein Ersuchen an die Polizei, präventiv tätig zu werden. Die Aussage der/des den Anruf entgegennehmenden Polizeibeamtin/Polizeibeamten als Zeugin/Zeuge in der Hauptverhandlung darüber, was im Rahmen des Hilfeersuchens geäußert wurde, oder der Mitschnitt dieses Anrufs sind verwertbare Beweismittel, auch wenn die Geschädigte selbst in der Hauptverhandlung von ihrem Zeugnisverweigerungsrecht Gebrauch macht.

Gibt es keine richterliche Zeugenvernehmung und kann die Staatsanwaltschaft auch nicht auf eine Spontanäußerung zurückgreifen, um einen Nachweis trotz Zeugnisverweigerung der Geschädigten zu führen, so besteht trotzdem noch kein Anlass, das Verfahren ohne Weiteres mangels Nachweises einzustellen.

Unter Umständen kann auf Zeug/inn/en aus dem Umfeld der Geschädigten zurückgegriffen werden. Gerade bei sehr jungen Geschädigten sind deren Eltern oft froh, Angaben dazu zu machen, was die Tochter ihnen erzählt hat und welche Verletzungen sie wann an ihr festgestellt haben. Auch die Aussage von Personen aus der Nachbarschaft, dem Freundes- oder Kollegenkreis kann die Ermittlungen weiterbringen und zusammen mit anderen Beweismitteln trotz fehlender Aussagebereitschaft des Opfers zu einem Nachweis führen.
Wenn es nun gelingt, trotz Widerstands des Opfers und gegen seinen erklärten Willen den Nachweis zu führen und den Beschuldigten anzuklagen, erfordert die Rücksicht auf das Opfer im Hinblick auf seine besondere Situation gewisse Vorsichtsmaßnahmen. In den Akten und in der Anklageschrift ist festzuhalten, dass die Anklage sich nicht auf die Aussage des Opfers stützen konnte, sondern dass das Opfer im Gegenteil versuchte, den Täter zu entlasten, dass der Beweis aber dennoch geführt wurde. Dies soll dem Opfer nach Möglichkeit ersparen, dass es durch den Täter für die Anklageerhebung verantwortlich gemacht wird und dafür büßen muss.

Wenn nach einer Trennung eine Gefährdung der Geschädigten für den Fall zu besorgen ist, dass der Täter ihren neuen Aufenthaltsort erfährt, sind in der gesamten Strafakte und in der Anklageschrift die Angabe ihrer Anschrift und alle Hinweise darauf zu tilgen. ${ }^{9}$ Die Ladung der Geschädigten zur Gerichtsverhandlung wird, wenn sie keine anwaltliche Vertretung hat, über die die Ladung erfolgen kann, durch die Staatsanwaltschaft bewirkt. Die Staatsanwaltschaft bewahrt die aktuelle Adresse bis zur Beendigung der Gefährdung sicher im Tresor der Behörde auf. ${ }^{10}$ Wenn dem Opfer diese Möglichkeit frühzeitig aufgezeigt wird, kann es hierdurch zur Aussage motiviert werden. Hinweise auf die Möglichkeit der Nebenklage und den Anspruch auf anwaltliche Vertretung während des gesamten Verfahrens, auch schon im Vorfeld der Anklageerhebung ${ }^{11}$, stärken die Position eines Opfers häuslicher Gewalt und verbessern die Chancen dafür, dass die Geschädigte bei ihrer Aussagebereitschaft bleibt.

Natürlich lässt sich nicht in jedem Fall ein Nachweis führen. Aber auch wenn das Verfahren eingestellt wird, werden in den Sonderdezernaten Besonderheiten beachtet.

Es gilt hier zum einen zu vermeiden, was früher dazu geführt hat, dass Opfer in Wiederholungsfällen noch weniger aussagebereit waren als zuvor. Bei einer Verfahrenseinstellung darf bei dem Opfer nämlich nicht der Eindruck entstehen, dass man ihm künftig nicht mehr glauben würde, und dass es ab jetzt selbst Schuld wäre, wenn wieder etwas passierte. Es ist zwar menschlich nachvollziehbar, dass Ermittlungspersonen über den Rückzug des Opfers, dem sie doch hatten helfen wollen, enttäuscht sind. Dies darf aber dem Opfer nicht vermittelt werden. Wir wollen ja in künftigen Fällen - die es aller Wahrscheinlichkeit nach geben wird - eine Aussage des Opfers fördern. Ein angemessener innerer Abstand der ermittelnden Personen zu der Sache ist hier erforderlich, ebenso wie das Wissen, dass die in einer Gewaltbeziehung verfangenen Opfer für Außenstehende meist völlig unlogisch und unverständlich reagieren, dass sie für eine Trennung fast immer mehrere Anläufe benötigen und dass dieser vorliegende Fall vielleicht einer der notwendigen ersten Schritte auf dem Weg zur endgültigen Trennung war.

8 Angaben, die gegenüber anderen Zeug/inn/en, beispielsweise der Mutter, der Freundin, dem Vermieter, gemacht wurden, sind davon nicht betroffen.

$9 \S 68$ Abs. 2 Abs. 5 StPO.

$10 \S 68$ Abs. 4 StPO.

$11 \S 397$ a Abs. 1, Abs. 2 i.V.m. $\S \S 395$ StPO; $\$ 406 g$ Abs. 1, Abs. 3 StPO. 
Weiter hat es sich bei Verfahrenseinstellungen, die wegen des Zeugnisverweigerungsrechts der Geschädigten erfolgen müssen, als sinnvoll erwiesen, die Geschädigte und den Beschuldigten über die Möglichkeit einer Wiederaufnahme des Verfahrens zu informiert. Eine Einstellung gemäß $\mathbb{S} 153$ Absatz $1^{12}, 170$ Absatz $2^{13}$ StPO bewirkt keinen Strafklageverbrauch ${ }^{14}$, sodass bei Änderung der Beweislage, insbesondere wenn sich das Opfer zu einem späteren Zeitpunkt zur Aussage entschließt, das Verfahren wieder aufgenommen und auch angeklagt werden kann, solange noch keine Verjährung eingetreten ist. ${ }^{15}$ Dies wird auch tatsächlich in der Praxis so umgesetzt. Manchmal erst nach Jahren ist das Opfer an einem Punkt angelangt, an dem es verstanden hat, dass der Täter sich nicht mehr bessern wird. Und dass die Gewalt auch nicht noch viel schlimmer werden kann, als sie jetzt schon ist. Wenn sich das Opfer nun von dem gewalttätigen Partner trennt, folgt oft eine umfassende Aussage, auch über Vorfälle aus der Vergangenheit. Hier können dann die früher angelegten Verfahrensakten, die nach der Einstellung mangels Nachweises ins Archiv gelangten, wieder aufgenommen und zur Grundlage der Beweisführung gemacht werden. Die zu den damaligen Tatzeiten erhobenen Beweise sind jetzt wichtige Hilfsmittel: Die Geschädigte ist nach Jahren zwar aussagewillig, aber kann aufgrund der Vielzahl der ähnlichen Vorfälle oft Taten und Tatzeiten, Begehungsweisen und Umstände nicht mehr konkret und unterscheidbar benennen. Wenn ihr dann aber Vorhalte aus den alten Verfahrensakten gemacht werden können, kehrt die konkrete Erinnerung zurück, sodass die Taten anklagefähig beschrieben sind und abgeurteilt werden können.
In zahlreichen Sonderdezernaten bundesweit wird täglich dieselbe Erfahrung gemacht: Mit besonderem Hintergrundwissen, spezieller Ermittlungstaktik und vorausschauender Vorgehensweise lassen sich die Herausforderungen, vor die uns die Fälle häuslicher Gewalt stellen, meistern. Der Arbeit in den allgemeinen Abteilungen der Staatsanwaltschaften ist dagegen das zur Verfolgung häuslicher Gewalt notwendige Durchsetzen der Strafverfolgung gegen den Willen des Opfers fremd. Dort ist man eher daran gewöhnt, unrealistische Erwartungen von Geschädigten an eine möglichst hohe Bestrafung zurückzuweisen. Auch der Blick dafür, dass zunächst eher harmlos erscheinende Sachverhalte unter Umständen tiefe Abgründe und Hochrisikofälle verbergen können, entsteht erst während der intensiven Beschäftigung mit Fällen häuslicher Gewalt.

Unter Menschenrechtsgesichtspunkten, aber auch schon aus wirtschaftlichen Erwägungen heraus, ${ }^{16}$ kommt ein Staat nicht umhin, effektive Maßnahmen zur Bekämpfung von häuslicher Gewalt zu ergreifen. Dabei stellt eine konsequente und effektive Strafverfolgung einen wichtigen Baustein dar. Sie ist ohne Sonderdezernate bei den Staatsanwaltschaften nicht zu leisten.

12 Einstellung wegen geringer Schuld.

13 Einstellung mangels ausreichenden Nachweises.

14 Meyer-Goßner, Lutz, StPO, 53. Aufl. 2010, § 153 StPO Rn. 37, § 170 Rn. 9.

15 Vorsätzliche Körperverletzung verjährt erst nach fünf, gefährliche Körperverletzung nach zehn, Vergewaltigung nach 20 Jahren.

16 Häusliche Gewalt ist ein erheblicher Kostenfaktor, vgl. beispielsweise Schätzung des, National Center for Injury Prevention and Control' für die USA auf 5,8 Mrd. US-Dollar jährlich, <www.cdc.gov/ violenceprevention/pdf/IPVBook-a.pdf> (Zugriff: 16.1.2013).

\section{Qualifizierung forensischer Sachverständiger durch die Psychotherapeutenkammern}

\section{Marion Schwarz}

Diplom-Psychologin, Kinder- und Jugendlichenpsychotherapeutin, Psychologische Psychotherapeutin, Sachverständige für Familienrecht, Bad Schwalbach

Die Heilberufegesetze der Bundesländer sehen für die Kammern auch die Aufgabe vor, Sachverständigenlisten zu führen. Daher haben die Landespsychotherapeutenkammern vor fünf Jahren eine Arbeitsgruppe gegründet, die Kriterien für die Anerkennung und Fortbildung von forensischen Sachverständigen aus dem Mitgliederbereich der Psychotherapeut/inn/en ${ }^{1}$ entwickeln sollte. Diese AG erarbeitete ein Modell für die qualifizierte Fortbildung/Weiterbildung von Sachverständigen sowie Übergangsbestimmungen für die Anerkennung bereits tätiger Sachverständiger, jeweils bezogen auf ein Rechtsgebiet.

Die einzelnen Landeskammern sind nunmehr dabei, diese Musterrichtlinie durch jeweils eigene Fort- oder Weiterbildungsordnungen umzusetzen. ${ }^{2}$

\section{Modulare Fort-/Weiterbildung}

Die Fortbildung ist modular aufgebaut. Zunächst sollen über ein Grundlagenmodul allgemeine Rechtsgrundlagen und die Aufgaben als Sachverständige vermittelt werden, sowie spezifische psychologische Kenntnisse.

Darauf aufbauend werden verschiedene Spezialisierungsmodule angeboten:

- zum Strafrecht und Strafvollstreckungsrecht,

- zur Glaubhaftigkeit der Zeugenaussage,

vur Prognosebeurteilung,

- zum Familienrecht,

- zum Sozialrecht.

1 Psychologische Psychotherapeut/inn/en und Kinder- und Jugendlichenpsychotherapeut/inn/en.

2 Psychotherapeutenkammer Hessen-LPPKJP, Psychotherapeutenkammer Rheinland-Pfalz, Ostdeutsche PsychotherapeutenkammerOPK, Psychotherapeutenkammer NRW, Psychotherapeutenkammer Niedersachsen, Psychotherapeutenkammer Bremen, Psychotherapeutenkammer Bayern, Psychotherapeutenkammer Saarland. 